\title{
A MODEL FOR THE FREE-SURFACE FLOW DUE TO A SUBMERGED SOURCE IN WATER OF INFINITE DEPTH
}

\author{
J.-M. VANDEN-BROECK ${ }^{1}$
}

(Received 3 November 1995; revised 27 September 1996)

\begin{abstract}
We consider a free-surface flow due to a source submerged in a fluid of infinite depth. It is assumed that there is a stagnation point on the free surface just above the source. The freesurface condition is linearized around the rigid-lid solution, and the resulting equations are solved numerically by a series truncation method with a nonuniform distribution of collocation points. Solutions are presented for various values of the Froude number. It is shown that for sufficiently large values of the Froude number, there is a train of waves on the free surface. The wavelength of these waves decreases as the distance from the source increases.
\end{abstract}

\section{Introduction}

Free-surface flows due to a submerged source have been studied by many authors ([1]-[5], [7], [9]-[11]).

Here we consider the free-surface flow due to a submerged source in water of infinite depth. Tuck and Vanden-Broeck [7], showed that there are two possible flows. One is characterized by a cusp on top of the source (see Figure 1a) and the other by a stagnation point on top of the source (see Figure 1b).

The flow sketched in Figure la was solved by Tuck and Vanden-Broeck [7]. They showed that there is a unique solution corresponding to a value of the Froude number $F$ equal to 3.55 . Here the Froude number is defined by

$$
F=\left(\frac{m^{2}}{g h^{3}}\right)^{1 / 2}
$$

where $m$ is the strength of the source, $h$ the submergence of the source beneath the undisturbed level of the free surface and $g$ the acceleration of gravity.

\footnotetext{
'Department of Mathematics and Center for the Mathematical Sciences, University of WisconsinMadison, WI 53706, USA

(C) Australian Mathematical Society, 1998, Serial-fee code 0334-2700/98
} 


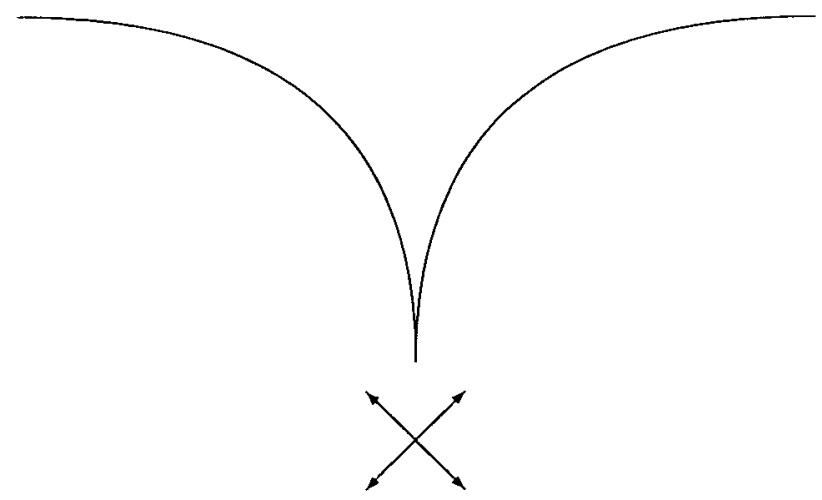

(a)

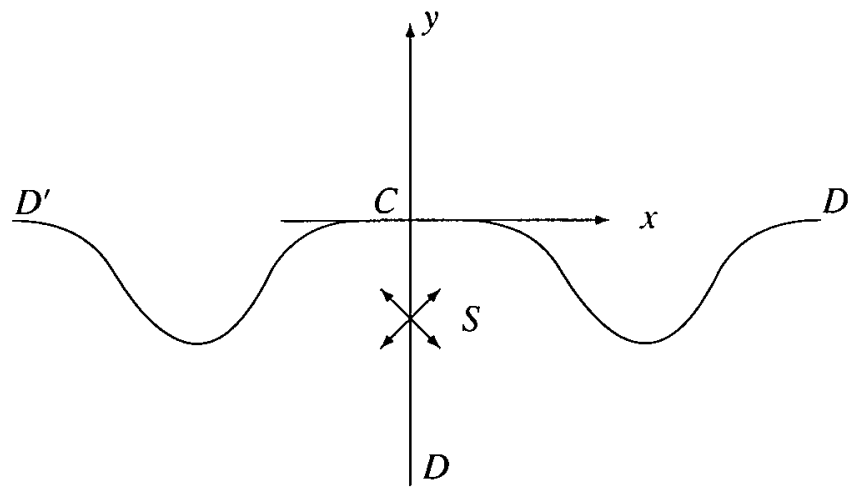

(b)

FIGURE 1. Sketch of free-surface flows due to a submerged source in water of infinite depth. There is a cusp on top of the source in Figure la and a stagnation point in Figure lb.

The flow sketched in Figure 1b has a longer history. Peregrine [5] obtained four terms in an expansion with respect to a parameter physically equivalent to $F^{2}$. VandenBroeck, Schwartz and Tuck [11], were able to continue the expansion indefinitely and showed that it has a zero radius of convergence. They then summed this divergent expansion by using a procedure due to Shanks [6]. However the "solutions" obtained by this procedure are not physical because they have a finite jump discontinuity at a particular point on the free-surface.

Such "solutions" with jump discontinuities were also obtained for a two-dimensional model for stern waves [11], [12]. For that problem, it was shown that the unphysical jump is related to the existence of waves of exponentially small amplitude on the free- 
surface as the Froude number approaches zero. Continuous solutions (with waves on the free-surface) were obtained numerically by using a portion of the discontinuous solutions as a starting point in an iterative procedure ([11], [12]) and analytically by a series completion procedure [8].

It is therefore natural to ask if the jump discontinuity for the source problem is also related to exponentially small waves. This is not obvious because the flow in Figure $1 \mathrm{~b}$ approaches zero in the far field whereas the stern flows of the previous paragraph approach a uniform stream in the far field. The difference between these two cases can easily be understood by recalling that the wavelength of linear gravity waves is proportional to the square of the phase velocity. Therefore if we apply the linear theory to the flow in the far field for the source problem, we find that the wavelength of the waves approaches zero in the far field. Furthermore since the stagnation level is the highest level on the free-surface, it is also necessary for the amplitude of the waves to approach zero in the far field. These are very strong requirements on the train of waves. On the other hand, linear theory applied to the far field of the stern flow simply predicts a train of waves of constant amplitude and nonzero constant wavelength (this is confirmed by the calculations in [8], [11] and [12]).

The previous discussion suggests that the source problem of Figure $1 \mathrm{~b}$ should be easier to solve in water of finite depth. We then have again a uniform stream in the far field and linear theory predicts a uniform train of waves. Indeed the problem in finite depth was solved in ([2], [4], [9]) and the calculations in [4], [9] show that there is a uniform train of waves in the far field. Furthermore it is shown in [4], that the waves are exponentially small as the Froude number approaches zero.

Hocking and Forbes [1] used a series truncation procedure to solve the flow configuration of Figure $1 \mathrm{~b}$. They present numerical solutions, but their scheme fails to converge for $F>1.42$. The question of the existence of solutions for $F>1.42$ is open.

In this paper we shed some light on this open question by considering a simplified formulation of the flow of Figure $1 b$ in which the free-surface condition is linearized around the rigid-lid solution. We present a series-truncation procedure which enables us to compute solutions for all values of $F$, including values larger than 1.42. For $F$ small, our solutions are very close to those of Hocking and Forbes. However for larger values of $F$, it is found that there is a train of waves on the free-surface. This suggests that the jump discontinuity obtained by using the Shanks procedure in [11] is associated with exponentially small waves, as in the stem-flow problem. The wavelength of the waves decreases with distance from the source in accordance with the linear theory.

The problem is formulated in Section 2 and the numerical results are presented in Section 3. Some concluding remarks on the connection between our linear model and the complete nonlinear problem are presented in Section 4. 


\section{Formulation}

We consider a fluid flow due to a source $S$ of strength $m$. We assume that the fluid is inviscid and incompressible and that the flow is irrotational. The flow domain is bounded above by a free surface $D^{\prime} C D$. The point $C$ on the free-surface and just above the source is a stagnation point (see Figure $1 \mathrm{~b}$ ). We define dimensionless variables by choosing $\left(m^{2} /\left(8 \pi^{2} g\right)\right)^{1 / 3}$ as the unit length and $(m g / \pi)^{1 / 3}$ as the unit velocity. We choose cartesian coordinates with the origin at $C$. We write

$$
z=x+i y,
$$

and we introduce the complex potential function

$$
f=\phi+i \psi
$$

and the complex velocity

$$
\zeta=\frac{d f}{d z}=u-i v .
$$

Here $u$ and $v$ are the horizontal and vertical components of the velocity. Without loss of generality we chose $\phi=0$ at the stagnation point $C$ and $\psi=0$ on the streamlines $D^{\prime} C D$ and $C S$. It follows from our choice of dimensionless variables that $\psi=-\pi$ on the vertical streamline $S D$.

The condition of constant pressure on the free-surface yields

$$
u^{2}+v^{2}+y=0
$$

Following Tuck and Vanden-Broeck [7], we map the flow domain into the half of the unit circle in the lower half $t$-plane by the transformation

$$
f=\log \frac{4 t}{(t+1)^{2}} .
$$

The point $D$ at infinity, the source $S$ and the stagnation point $C$ are mapped onto $t=-1, t=0$ and $t=1$ respectively. Thus the portion $C D$ of the free-surface is mapped onto the circular arc

$$
t=e^{i \sigma}, \quad-\pi<\sigma<0 .
$$

We seek $z$ as an analytic function of $t$. We denote the values of $z$ and of $\zeta$ on the free-surface by $Z(\sigma)=X(\sigma)+i Y(\sigma)$ and $\Sigma(\sigma)=U(\sigma)-i V(\sigma)$ respectively. Using (4), (6), (7) and the chain rule, we find that $Z(\sigma)$ and $\Sigma(\sigma)$ are related by

$$
\Sigma(\sigma)=\frac{\tan (\sigma / 2)}{Z_{\sigma}(\sigma)}
$$


Substituting (8) into (5) we obtain

$$
\frac{\tan ^{2}(\sigma / 2)}{X_{\sigma}^{2}(\sigma)+Y_{\sigma}^{2}(\sigma)}+Y(\sigma)=0
$$

The rigid-lid solution is obtained by replacing the free surface by the line $y=0$. The problem then has an exact solution, namely

$$
z_{0}(t)=-i b_{1} \frac{1-t}{1+t}
$$

where $b_{1}$ is a real constant. On the free surface, $(10)$ yields $Y_{0}(\sigma)=0$ and

$$
Z_{0}(\sigma)=X(\sigma)=-b_{1} \tan (\sigma / 2)
$$

Setting $t=0$ into (10), we find that the ordinate of the source is $-b_{1}$. From (9) and (10), we see that the rigid-lid solution is the solution of our problem in the limit as $b_{1} \rightarrow \infty$.

The basic assumption in this paper is to linearize the flow around the rigid-lid solution (10). Thus we write

$$
z(t)=z_{o}(t)+z_{1}(t)
$$

where $z_{1}(t)$ is assumed to be a small perturbation.

Substituting (12) into (9), and retaining only the terms linear in $z_{1}$ we obtain

$$
\frac{\sin ^{2} \sigma}{b_{1}^{2}}+\frac{4 \sin ^{2} \sigma \cos ^{2}(\sigma / 2)}{b_{1}^{3}} X_{1}^{\prime}+Y_{1}=0
$$

Following Tuck and Vanden-Broeck [7], we represent $z_{1}$ by the power-series expansion

$$
z_{1}=-\frac{i}{1+t} \sum_{n=2}^{\infty} b_{n} t^{n-1}
$$

We impose the conditions

$$
\sum_{n=2}^{\infty} b_{n}=0
$$

and

$$
\sum_{n=2}^{\infty}(-1)^{(n-1)}(2 n-3) b_{n}=0
$$


Relation (15) imposes a stagnation point at $C$ and (16) ensures that the free-surface condition is satisfied at physical infinity (see [7] for details). Finally we note that the ordinate $-b_{1}$ of the source is related to the Froude number $F$ defined in (1) by

$$
F^{2}=\frac{8 \pi^{2}}{b_{1}^{3}}
$$

This concludes the formulation of the problem. For a given value of $F$ (or equivalently $b_{1}$ ), we seek the coefficients $b_{2}, b_{3}, \ldots$ such that (13)-(16) are satisfied. This is achieved numerically in the next section.

\section{Numerical results}

We evaluate the coefficients $b_{2}, b_{3}, \ldots$ by collocation. First we truncate the infinite series in (14), after $N-1$ terms. The $N-1$ unknowns coefficients $b_{2}, b_{3}, \ldots, b_{N}$ are found by satisfying (13) at $N-3$ mesh points $\sigma=\sigma_{I}, \quad I=1, \ldots, N-3$ distributed between $-\pi$ and 0 . The last two equations are obtained by imposing the conditions (15) and (16). This system of $N$ linear algebraic equations with $N$ unknowns is solved numerically. The free-surface profiles are obtained by integrating numerically the identity $d z / d f=\zeta^{-1}$. In all the results presented, only half of the free-surface is shown (the other half can be obtained by symmetry).

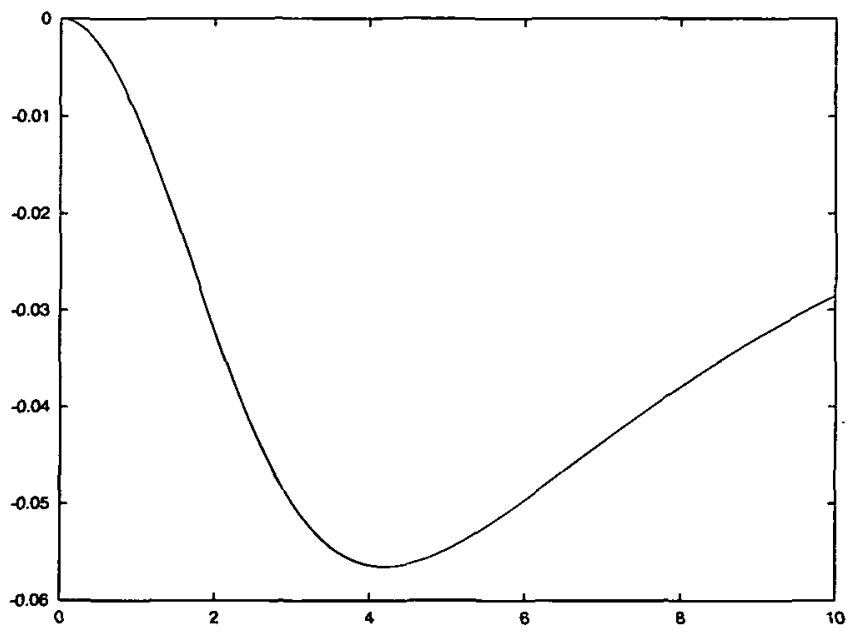

FIGURE 2. Free-surface profile for $F=1$. This solution was computed with $N=158$.

In preliminary computations, we used equally-spaced mesh points $\sigma_{l}$, namely

$$
\sigma_{l}=-\frac{\pi}{N-2} I, \quad I=1, \ldots, N-3 .
$$


For a given value of $N$, the scheme converged in a few iterations. For $F<1.4$, the profiles are essentially waveless (see Figure 2). However for larger values of $F$, there are waves on the free-surface both near the stagnation point $C$ and in the far field (see Figure 3). We found that these "solutions". are dependent on the value of $N$ chosen, and no convergence was observed as $N$ increases. In particular the amplitude of the waves varies with $N$. This is illustrated in Figure 3, where we present two "solutions" for $F=1.9$ computed with $N=358$ (Figure 3a) and $N=258$ (Figure 3b). The "solution" in Figure 3a is almost waveless in the far field whereas the "solution" in Figure $3 \mathrm{~b}$ has a train of waves both in the far field and near the stagnation point. Because of this dependence on $N$, these "solutions" should be considered as spurious numerical solutions.

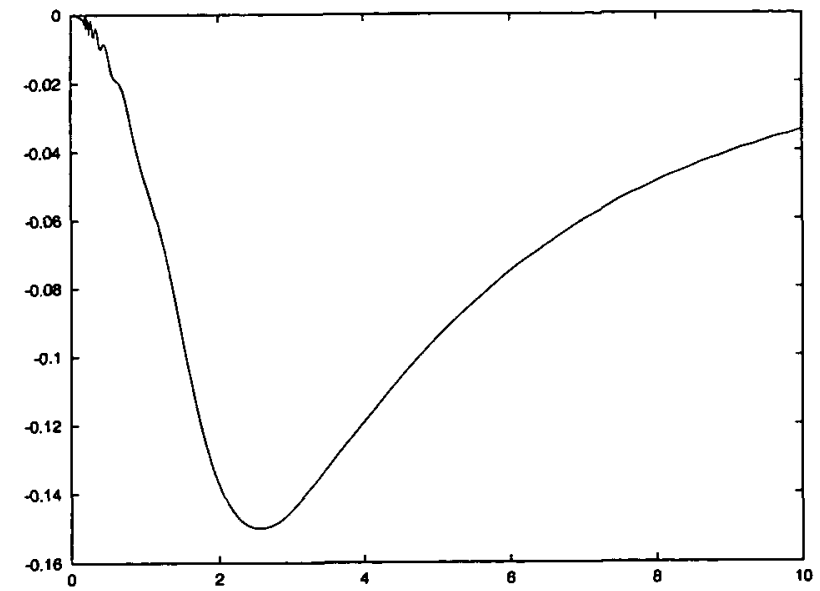

(a)

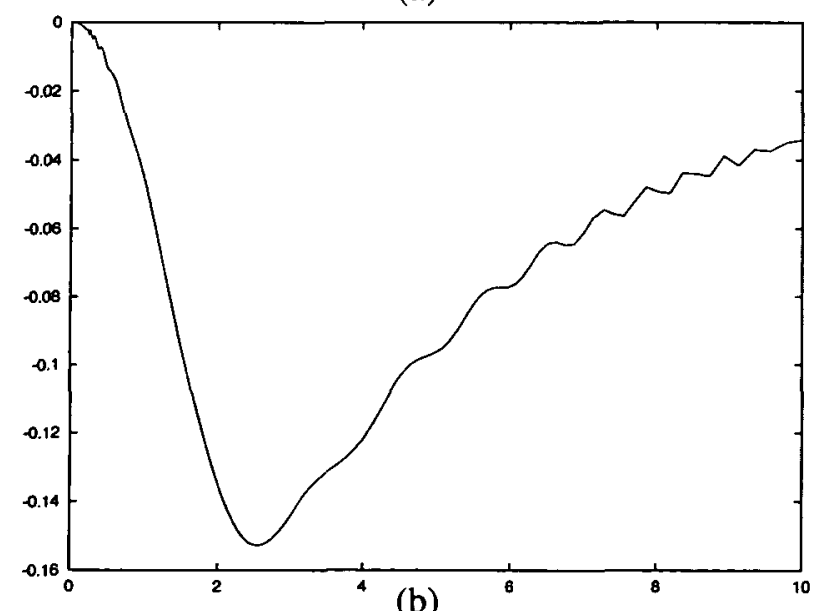

(b)

FigURE 3. Spurious numerical free-surface profiles for $F=1.9$ with a) $N=358$ and b) $N=258$. 
After many different tries, we found that true numerical solutions (that is, solutions which converge as $N$ increases) can be obtained by using a slightly nonuniform distribution of mesh points. As we shall see, these solutions are waveless near the stagnation point but have a train of waves in the far field. Therefore the nonuniformity can be viewed as a technique to remove the oscillations near the stagnation point.

The modified distribution of mesh points is defined by

$$
\sigma_{l}=-\frac{\pi}{N-2} \alpha-\pi \frac{N-2-2 \alpha}{(N-2)(N-4)}(I-1), \quad I=1, \ldots, N-3
$$

where $\alpha$ is a parameter. For $\alpha=1,(19)$ reduces to (18). For $\alpha \neq 1$, the mesh spacing is not equal to $\sigma_{1}$, and $0, \sigma_{1}, \sigma_{2}, \ldots, \sigma_{N-3}$ form a set of points nonuniformly spaced near 0.

Our numerical procedure is the following. We start with a "solution" with $\alpha=1$ like the ones in Figure 3. We then repeat the calculations for different values of $\alpha$. We found that the amplitude of the oscillations on the free-surface (and especially those near the stagnation point) depends on $\alpha$. The numerical scheme has the property that there are particular values $\alpha^{*}$ of $\alpha$ for which the oscillations near the stagnation point disappear. The values of $\alpha^{*}$ depend on $N$ and $F$. We found that the solutions corresponding to $\alpha^{*}$ are true numerical solutions. In other words, for a given value of $F$, the solutions corresponding to $\alpha^{*}$ are independent of $N$, for $N$ sufficiently large.

We now illustrate the above discussion by presenting numerical solutions for $F=$ 1.9. We performed calculations with $N=158, N=258$ and $N=358$. The corresponding values of $\alpha^{*}$ are 1.3,1.18 and 1.13. The free-surface profiles are shown in Figures $4 \mathrm{a}-4 \mathrm{c}$. For a fixed value of $x$, the solutions converge as $N$ increases. For example, the three profiles of Figure 4 coincide for $x<7$ (this is to be contrasted with the behaviour of the "solutions" in Figure 3).

It should be noted that the waves in Figure $4 \mathrm{a}$ are "damped" in the far field and almost of zero amplitude for $x>9$. However, Figures $4 b$ and $4 c$ show that this "damping" moves to larger and larger values of $x$ as $N$ is increased. In fact a comparison of the profiles of Figure 4 strongly suggests that the waves in the far field approach a train of waves of constant amplitude as $x \rightarrow \infty$. The wavelength of this train of waves decreases as $x$ increases.

Next we present in Figure 5 a solution for $F=2.1$. This solution was obtained with $N=158$ and $\alpha=\alpha^{*}=2.07$. The wave is damped in the far field but the damping can be pushed to larger values of $x$ by increasing $N$.

Finally let us mention that as $F$ decreases our solutions merge continuously into the essentially waveless solutions described in Hocking and Forbes [1] and in Figure 2. 


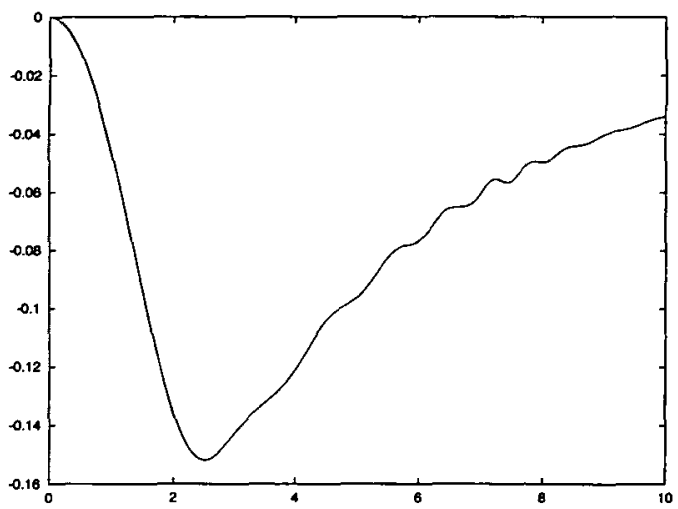

(a)

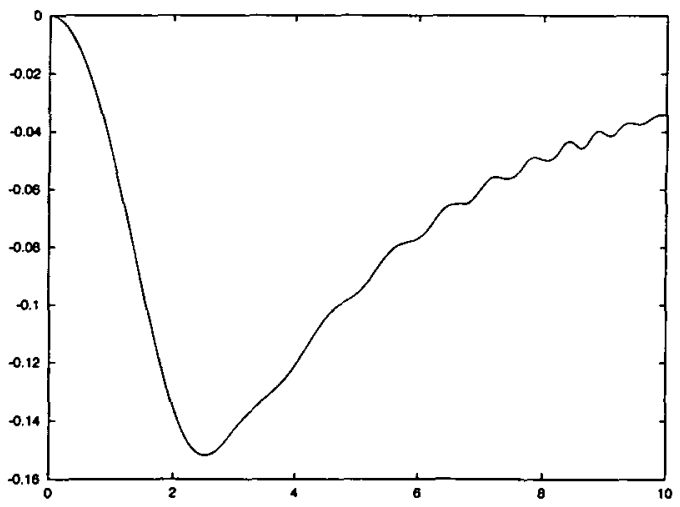

(b)

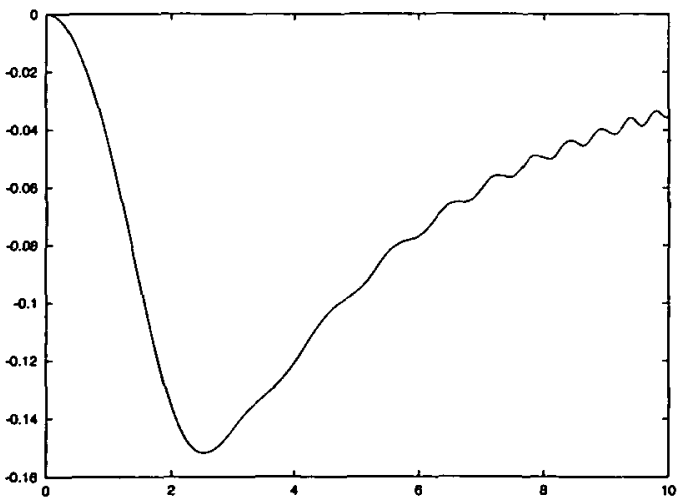

(c)

FIGURE 4. Free-surface profiles for $F=1.9$. These solutions were computed with a) $N=158$, $\alpha^{*}=1.3$, b) $N=258, \alpha^{*}=1.18$ and c) $N=358, \alpha^{*}=1.13$. 


\section{Conclusions}

We have computed solutions for a linearized model of a source submerged below a free-surface. The solutions were obtained by using a series truncation procedure with a slightly nonuniform distribution of mesh points. Our solutions are characterized by a train of waves of constant amplitude in the far field. The wavelength of the waves approaches zero as $|x| \rightarrow \infty$ in accordance with linear theory. By analogy with the stern flow and other problems described in the introduction, we expect that the waves are exponentially small as $F \rightarrow 0$. These are already too small to be seen in Figure 2 . We also expect the discontinuous solutions obtained in [11] to agree with our solutions between the stagnation points and the discontinuities.

It is worthwhile to mention that solutions with a train of waves of constant amplitude in the far field are not possible for the complete nonlinear problem. As $|x| \rightarrow \infty$, $u \rightarrow 0$ and $v \rightarrow 0$. Therefore (5), implies that $y$ on the free-surface must approach zero as $|x| \rightarrow \infty$. This eliminates waves of constant amplitude (such waves would ultimately reach their "breaking height" as $x$ increases). Therefore the solutions of Figures 4 and 5 cannot exist for the complete nonlinear problem.

There are, however, two ways in which our solutions can approximate nonlinear solutions. First in a real fluid, viscosity will ultimately damp the train of waves in the far field and a real viscous solution might in fact look like the solution in Figure $4 \mathrm{a}$ (that is, profiles with a damped train of waves in the far field). Secondly if $F$ is small enough, then the waves are so small that they can be neglected. For example we have repeated the nonlinear calculations of Hocking and Forbes [1] for $F=1$ and found that their profile coincides with our solution in Figure 2 within graphical accuracy.

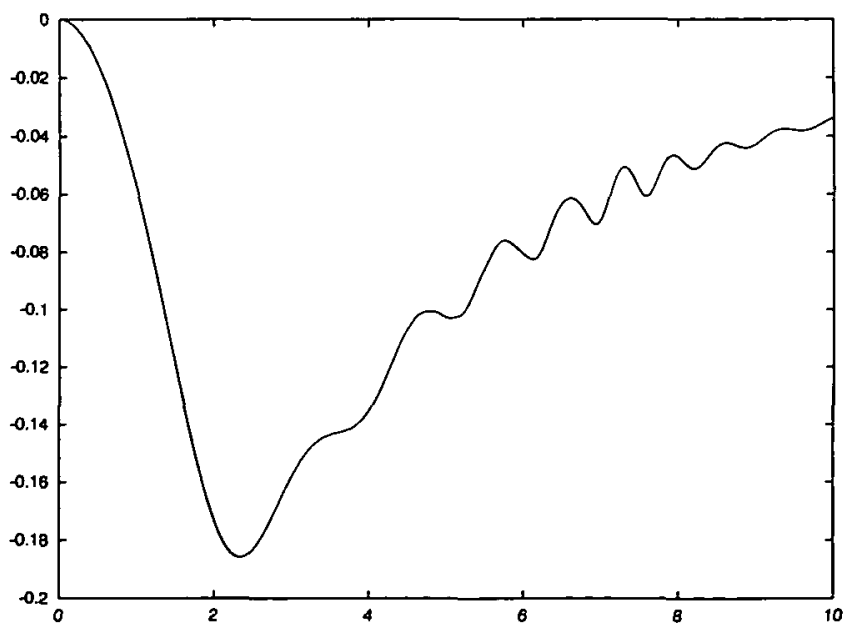

FlGURE 5. Free-surface profile for $F=2.1$. This solution was computed with $N=158$ and $\alpha^{*}=2.07$. 


\section{Acknowledgement}

This work was supported in part by the National Science Foundation, and the Lady Davis Foundation.

\section{References}

[1] G. C. Hocking and L. K. Forbes, "A note on the flow induced by a line sink beneath a free surface", J. Aust. Math. Soc. Ser: B 32 (1990) 251-260.

[2] G. C. Hocking and L. K. Forbes, "Subcritical free-surface flow caused by a line source in a fluid of finite depth", J. Eng. Math. 26 (1992) 455-466.

[3] H. Mekias and J.-M. Vanden-Broeck, "Supercritical free-surface flow with a stagnation point due to a submerged source", Phys. Fluids A 1 (1989) 1694-1697.

[4] H. Mekias and J.-M. Vanden-Broeck, "Subcritical flow with a stagnation point due to a source beneath a free surface", Phys. Fluids A 3 (1991) 2652-2658.

[5] D. H. Peregrine, "A line source beneath a free surface", Rep. no 1248, Math. Res. Cent. (University of Wisconsin, Madison, 1972).

[6] D. Shanks, "Non-linear transformations of divergent and slowly-convergent sequences", J. Math. Phys. 34 (1955) 1-42.

[7] E. O. Tuck and J.-M. Vanden-Broeck, "A cusp-like free-surface flow due to a submerged source or sink", J. Aust. Math. Soc. Ser: B 25 (1984) 443-450.

[8] J.-M. Vanden-Broeck, "Nonlinear free-surface flows past two-dimensional bodies", in Advances in Nonlinear Waves, Vol II, (ed. L. Debnath) (Boston, Pitman, 1985).

[9] J.-M. Vanden-Broeck, "Waves generated by a source below a free surface in water of finite depth", J. Eng. Math. 30 (1996) 603-609.

[10] J.-M. Vanden-Broeck and J. B. Keller, "Free surface flow due to a sink", J. Fluid Mech. 175 (1987) 109-117.

[11] J.-M. Vanden-Broeck, L. W. Schwartz and E. O. Tuck, "Divergent low-Froude-number series expansion of nonlinear free-surface flow problems", Proc. R. Soc. Lond. A 361 (1978) 207-224.

[12] J.-M. Vanden-Broeck and E. O. Tuck, "Computations of near-bow or stem flows, using series expansions in the Froude number", Proc. 2nd Int. Conf. Num. Ship Hydrodynamic, Berkeley, Calif. (1977) 371-381. 